fu, per Oakeshott, un precursore dello 'scientismo' in quanto pensava che la scienza avrebbe liberato l'uomo dalla superstizione e creato una "scienza del governo" che avrebbe applicato "il metodo scientifico al diritto, unito legge e scienza, scoperto qualche mezzo per misurare accuratamente le soddisfazioni politiche" (p. 274). Ma se queste erano le sue ambizioni, sintetizzabili nel credere che nell'ambito delle scienze sociali fosse necessario ripartire da zero ed elaborare un codice: "qualcosa di creato, organizzato e definito, e perciò distinto da ciò che era invece meramente cresciuto" (p. 274), Bentham non aveva le risorse intellettuali per portare a compimento un simile radicale ed originale progetto. Come quei "despoti benevolenti" che furono i philosophes, rimase così a metà strada e il suo odio verso la Common Law gli impedì di capire che anche "la legge deve cambiare" (p. 275). E questo avvenne perché in lui non c'era niente di originale e quindi, in sostanza, perché aveva anche scarsa attitudine per la filosofia. Tant'è che anche riguardo a quell'utilitarismo di cui viene considerato il moderno rifondatore, il suo pensiero diventa confuso e contraddittorio allorché, per quanto riguarda il concetto di utilità, si "avventura oltre ciò che altri avevano pensato" (p. 281). E questo perché quantunque avesse per tutta la vita parlato di "princìpi primi", non avendo un vero interesse per il pensiero speculativo, Bentham "non riuscì mai a considerare con attenzione altro che cose secondarie e derivate" (p. 284).

Come dire che se la filosofia è tutto sommato una pratica innocente ed inutile, un diletto per gentiluomini, una mezza filosofia è invece qualcosa che in genere è destinata a rivelarsi dannosa.

\title{
OAKESHOTT E IL RAZIONALISMO IN POLITICA
}

\author{
di Giovanni Giorgini*
}

L'attacco al razionalismo in politica costituisce il fulcro degli interessi teorici, e delle preoccupazioni pratiche, di Oakeshott negli anni Cinquanta. Con il termine razionalismo egli intende il supposto uso

* Università di Bologna. 
senza vincoli e senza pregiudizi della ragione e la fede che in questo modo si arriverà a un sicuro miglioramento della condizione umana. Nella sua ricostruzione, il primo autore a proporre l'uso della ragione, acuminata dal metodo induttivo e purificata da pregiudizi, come strumento della conquista della natura da parte dell'uomo fu Francis Bacon; una ragione strumentale che aveva fatto tabula rasa dei pregiudizi derivanti dalla società, dal principio di autorità, dalle opinioni comuni (i vari tipi di idola). L'applicazione di questa visione della ragione intesa come strumento alla politica induce a credere che essa possa agire prescindendo dalle circostanze, per esempio delineando in astratto uno Stato ideale che sarà poi compito del governo realizzare nella pratica: è, questa, la "politica della perfezione", che cerca di realizzare il Paradiso in terra e richiede grandi sacrifici agli uomini, mobilitandoli tutti verso una meta. Si tratta di una visione molto simile a quella che Hayek definiva "abuso della ragione", tipico della "mentalità ingegneristica" e Popper chiamava "ingegneria sociale utopica". Oakeshott attacca sia il fondamento teorico sia le conclusioni pratiche del Razionalismo.

Oakeshott intende mostrare la fallace epistemologia che si trova alla base del collettivismo e della pianificazione centrale. Egli mostra, infatti, che il Razionalismo si fonda su di una visione erronea della mente umana così come su di un'immagine impoverita della conoscenza. È un errore considerare 'conoscenza' solamente la conoscenza tecnica, che può essere espressa in regole e può essere insegnata e appresa (come la dimostrazione di un teorema o il Codice della Strada); vi è anche un altro genere di conoscenza, che Oakeshott definisce 'pratica' o 'tradizionale', che può essere acquisita solo attraverso l'uso e l'esperienza. È questo tipo di conoscenza che è alla base di attività umane come la politica e la cucina. Non si diventa buoni cuochi semplicemente leggendo i libri di cucina, che possono sì insegnare la tecnica culinaria ma non creare il gusto; solo il cuoco che ha fatto apprendistato ed è dotato di esperienza sa quanto sale aggiungere quando la ricetta indica "q.b.". Si noti che questa visione è sia una riproposizione della nozione aristotelica di phronesis - quella prudenza che consente di individuare la giusta medietà evitando l'errore (e il vizio) dei due estremi - sia una polemica contro l'idea allora dominante in filosofia, di stampo neopositivistico, secondo cui l'unica conoscenza sia quella che può essere formulata esplicitamente. In questa critica al 
Neopositivismo, all'idea che il compito della filosofia sia quello di formulare e testare ipotesi che siano empiricamente verificabili16, Oakeshott è accomunato a pensatori come Michael Polanyi, Gilbert Ryle e Friedrich von Hayek. Negli stessi anni, infatti, Polanyi, in The Logic of Liberty e in Personal Knowledge ${ }^{17}$, parlava dell'esistenza di una "dimensione tacita" e di una "conoscenza inespressa". Hayek, partendo dal postulato della razionalità limitata e rimarcando 'l'inevitabile ignoranza' che contraddistingue tutti gli esseri umani e che rende impossibile un'efficiente pianificazione centrale, attaccava il costruttivismo socialista. Ryle, infine, in The Concept of Mind"1 , distingueva tra "knowing how" e "knowing that", sostenendo che solo il secondo tipo di conoscenza può essere appreso senza bisogno di esperienza.

Nella visione di Oakeshott il razionalismo è nemico dell'autorità e del pregiudizio e, in generale, del mistero, perché ambisce a portare ordine e chiarezza in tutte le cose. Per mostrare la fallacia teorica del pensare che la ragione possa operare nel vuoto, senza interferenze da parte delle opinioni della società e delle circostanze storiche, Oakeshott, nel saggio La condotta razionale, ricorre all'esempio del progetto di alcuni designers Vittoriani di trovare un "abbigliamento razionale" per le donne che andavano in bicicletta. Essi cercavano la razionalità e la perfezione in assoluto ma conclusero con ciò che era razionale e adeguato alle convenzioni dell'epoca: non inventarono i calzoncini corti, gli shorts, bensì dei pantaloni lunghi e aderenti con sopra una gonna al ginocchio per ingentilire l'aspetto troppo maschile dell'abbigliamento: i bloomers. La ricerca della perfezione in politica, che considera 'razionale' solo la condotta deliberatamente diretta al raggiungimento di uno scopo formulato precedentemente e astraendo dalle circostanze, è un'illusione. Si tratta, però, di un'illusione pericolosa perché, quando si cerca di metterla in pratica, porta inevitabilmente al sogno del controllo assoluto della società: "l'unione di sogno e governo genera la tirannia". Un errore teorico si trasforma in un incubo, una volta realizzato nella pratica.

\footnotetext{
${ }^{16} \mathrm{Si}$ veda il classico A.J. Ayer, Language, Truth, and Logic, New York, Dover Publication, 1936.

${ }^{17}$ Rispettivamente: Chicago, The University of Chicago Press, 1951, e Chicago, The University of Chicago Press, 1958.

${ }^{18}$ London, Hutchinson's University Library, 1949.
} 
Al Razionalismo, alla sua fede nella verità e nella perfezione, Oakeshott contrappone una visione scettica della conoscenza, che fa da fondamento a una concezione liberale dello Stato nella quale hanno un ruolo centrale le nozioni di 'tradizione' e di 'governo della legge' (Rule of Law). Egli fa propria la visione che si ritrova nell'Illuminismo scozzese e nel fondatore della Scuola Austriaca dell'economia, Carl Menger, ripresa in quegli stessi anni da Hayek, secondo cui lo Stato moderno e le istituzioni politiche sono il frutto di innumerevoli scelte umane, fatte in un lungo arco di tempo, ma non di uno specifico disegno umano. Quelle scelte non erano la risposta ad astratte credenze bensì a specifiche situazioni contingenti, si inserivano all'interno di una specifica tradizione di comportamento. L'idea che vi sia un 'architetto' alla loro base, qualcuno che si sia posto un obiettivo e si sia dedicato a quello scopo specifico, è una fallacia razionalistica. Nella realtà, la tradizione politica di una società fornisce delle 'indicazioni' (intimations) su come eliminare incoerenze al suo interno, per esempio dando il diritto di voto alle donne quando sia apparso evidente che esse hanno la stessa capacità politica degli uomini. Da ultimo, alla visione del Razionalista, che considera il governo un enorme serbatoio di potere che deve essere utilizzato per imporre agli altri la propria visione di una società perfetta, Oakeshott contrappone la visione del 'governo della legge', del rispetto di regole di condotta che consentono a ogni individuo di perseguire la propria personale immagine della felicità. Perché la ricerca della felicità è lo scopo della vita umana.

\section{OAKESHOTT E HAYEK}

di Alberto Mingardi*

Lo stesso titolo di questa silloge di Oakeshott, Razionalismo in politica, evoca la critica di Hayek al 'costruttivismo'. Il tema delle convergenze e differenze fra Hayek e Oakeshott è ormai un campo molto arato ${ }^{19}$. Secondo Kenneth Minogue, Hayek e Oakeshott erano "buoni

* Università IULM di Milano.

${ }^{19} \mathrm{Si}$ vedano, fra gli altri, S. COTELLESSA, Il ragionevole disaccordo: Hayek, Oakeshott 